\title{
Science spéculative et métaphysique des sciences de la nature chez Hegel
}

\author{
Emmanuel Renault*
}

Résumé : On soutient souvent que la philosophie hégélienne de la nature a pour objectif de remplacer les sciences empiriques par une science spéculative qui est une métaphysique plutôt qu'une sicence. Selon une telle interprétation, cette partie du système hégélien repose sur un non sens épistémologique. Cet article tente de montrer au contraire que le concept de science spéculative repose sur une critique de la métaphysique et que la philosophie de la nature développe une épistémologie de la métaphysique des sciences empiriques.

Mots clés : Science / Métaphysique / Logique / Physique newtonienne / Système

\begin{abstract}
Speculative science and metaphysics of natural sciences in Hegel.
It is often said that the purpose of Hegel's Philosophy of Nature is to replace empirical science by a speculative science which is metaphysics rather than science. According to such an interpretation, this part of the Hegelian system is based on an epistemological nonsense. This paper tries to prove, on the contrary, that the notion of speculative science supposes a criticism of the metaphysics and that the philosophy of nature develops an epistemology of the metaphysics of the empirical sciences.
\end{abstract}

Keywords: Science / Metaphysics / Logic / Newtonian physic / System

Les philosophies de Schelling et de Hegel se caractériseraient par une tentative visant à substituer une métaphysique aux sciences positives et c'est dans leurs Naturphilosophie que l'absurdité de ce projet apparaitrait le plus clairement puisqu'il conduirait ces philosophes à s'opposer aux savoirs positifs les plus solides, comme la mécanique newtonienne, et à opposer à de telles sciences des thèses douteuses ou franchement fausses. On sait que dans la Préface de la Phénoménologie de l'esprit, Hegel écrit que son "propos est de collaborer à ce que la philosophie se rapproche de la forme de la science - se rapproche du but qui est de pouvoir se défaire de son

* ENS LSH, 15 Parvis René Descartes, 69366 Lyon Cedex 07 
nom d'amour du savoir et d'être savoir effectif ${ }^{1}$ ». Cette volonté de faire de la philosophie une science ne consisterait en fait qu'en une revendication anachronique de la définition de la métaphysique comme reine des sciences. Une telle interprétation estelle acceptable? Il semble qu'elle s'applique effectivement à certaines des nombreuses versions de la philosophie schellingienne de la nature, et encore faudrait-il préciser et nuancer ${ }^{2}$ Pour ce qui est de Hegel, elle relève du pur contresens, d'une part, parce que le concept hégélien de science est solidaire d'une critique de la métaphysique, d'autre part, parce qu'il définit le projet d'une fondation spéculative des sciences positives qui respecte l'indépendance des sciences positives en tentant d'articuler deux types de scientificité

Pour faire ressortir les moments fondamentaux du rapport que Hegel prétend instituer entre science spéculative et science positive, la question de la métaphysique présente un double intérêt. Elle permet d'une part de préciser le sens du concept de science spéculative qui, on l'a dit, est solidaire d'une certaine critique de la métaphysique (1). Elle permet en outre de préciser la nature du rapport de la science spéculative et de la science empirique (2). Nous préciserons pour conclure la signification épistémologique de la thèse hégélienne suivant laquelle toute science à sa métaphysique (3).

Que le concept hégélien de science spéculative soit lié à une critique de la métaphysique est un fait peu contestable : dans la plupart des textes où Hegel présente le point de vue spécifique de la spéculation, il développe également une critique de la métaphysique. Ce qui est vrai de la préface de la première édition de la Logique est également vrai de l'introduction de la Logique et du concept préliminaire de l'Encyclopédie. Ce fait est-il insignifiant ou faut-il au contraire considérer que le concept hégélien de science est essentiellement lié à une certaine critique de la métaphysique? Pour répondre à cette question, précisons la nature de la critique hégélienne de la métaphysique.

Au vu des textes mentionnés, il apparait immédiatement que cette critique de la métaphysique ne consiste pas en un rejet définitif de la métaphysique mais en la dénonciation d'une certaine forme de métaphysique que Hegel nomme «l'ancienne

1 Préface de la Phénoménologie de l'esprit, trad. J.P. Lefebvre, GF, 1991, p. 39.

2 Voir à ce propos, F. Fischbach et E. Renault, "Présentation ", in Schelling, Introduction à l'esquisse d'un système de Philosophie de la nature, Le livre de poche, 2001.

3 Encyclopédie [cité Enc.], § 246, add., Werke [cité W.], Suhrkamp, Francfort, 1970, vol. 9, p. 20 : « la physique et la philosophie doivent travailler main dans la main ". 
métaphysique ", la métaphysique ayant cours " avant la philosophie kantienne " ". D'une part, Hegel soutient que l'ancienne métaphysique est définitivement périmée et qu'il ne servirait à rien de tenter de la réanimer ${ }^{5}$ Mais d'autre part, il déplore l'abandon de la métaphysique et il juge qu' « il est pour le moins étrange qu'un peuple perde sa métaphysique "; il parle également du "spectacle étrange d'un peuple cultivé dépourvu de métaphysique " ${ }^{6}$ Hegel s'engage donc dans un double mouvement de critique et de défense de la métaphysique qui explique que l'on ait pu interpréter la Logique soit comme une tentative visant à accomplir la métaphysique ${ }^{7}$, soit au contraire comme une critique radicale de la métaphysique ${ }^{8}$

Pour déterminer si la défense où la critique l'emporte, il n'est pas inutile de prendre le rapport de Hegel à Kant pour fil conducteur. Dans ces textes introductifs, la référence à Kant est centrale : Hegel soutient que "la métaphysique véritable [eigentliche Metaphysik] est la pure philosophie spéculative [ou la Logique] " et il rappelle que "la philosophie critique fit certes déjà de la métaphysique la logique ${ }^{10}$ ". Kant est ainsi présenté comme celui qui périme définitivement une certaine forme de métaphysique par sa théorie des antinomies ${ }^{11}$, et qui définit le projet d'une transformation de la métaphysique en logique. C'est bien le projet d'une logique transcendantale que Hegel reprend à son compte lorsqu'il écrit que sa Logique " correspond à la logique transcendantale ${ }^{12}$ " de Kant. Mais il reproche également à Kant d'avoir mis en œuvre ce projet de manière inadéquate, au sens d'une substitution de la logique à la métaphysique (donc comme une négation de la métaphysique) et non au sens d'une transformation de la métaphysique. Au sens des définitions traditionnelles de la métaphysique, la logique transcendantale kantienne ne peut plus être considérée

4 Science de la Logique, trad. P.-J. Labarrière et G. Jarczyk, Aubier Montaigne, 1972-1981, en trois volumes, t. I [cité $\log$ I, pp. 2 et 13 ; Encyclopédie, I - La science de la logique, trad. B. Bourgeois, Vrin, 1970, § 27.

$5 \log I$, p. 4 : « quand la forme substantielle de l'esprit est passée en une autre figure, il est définitivement vain de vouloir maintenir les formes de la culture antérieure ".

6 Ibid., pp. 2-3.

7 Par exemple, A. Doz, La logique de Hegel et les problemes traditionnels de l'ontologie, Vrin, 1987.

8 Par exemple, B. Longuenesse, Hegel et la critique de la métaphysique, Vrin, 1981.

$9 \log I$, p. 5.

10 Ibid., p. 21.

11 Ibid., p. 173, "ce sont elles qui ont provoqué l'effondrement de la métaphysique précédente"; Science de la Logique, t. III [cité Log III], p. 356 « toute la métaphysique d'antan (...) s'est trouvée jetée par-dessus les moulins ".

12 Ibid., p. 34. C'est de la logique objective qu'il s'agit, mais c'est précisément cette logique objective qui prend la place de toute la métaphysique, l’ontologie comme la métaphysique spéciale (p. 36). 
comme une métaphysique puisqu'elle n'énonce que les règles de la pensée pure des objets de l'expérience (des phénomènes) et non les règles de la pensée des objets en général (des objets en tant que chose en soi). Kant propose en ce sens de substituer le nom d'analytique de l'entendement pur à celui d'ontologie ${ }^{13}$ C'est précisément cette restriction qui est refusée par Hegel lorsqu'il déplore que, dans la transformation kantienne de la métaphysique en logique, la logique n'ait plus qu' " une signification essentiellement subjective " " dérée "sans nul égard à la signification métaphysique " ${ }^{15}$ Il s'agira de produire une logique transcendantale qui, en étudiant les formes de pensée dans lesquelles se donne le réel, étudie le réel lui-même. La Logique transcendantale sera alors métaphysique puisque par l'étude des formes de la pensée, elle nous fera accéder à ce que les choses sont en elles-mêmes, et non pas simplement à leur apparence phénoménale : " La logique coïncide par conséquent avec la Métaphysique, la science des choses, saisies en des pensées qui passaient pour exprimer les essentialités des choses " ${ }^{16}$ Il importe de comprendre que lorsque Hegel s'oppose à la restriction kantienne de la logique transcendantale, il défend la thèse suivant laquelle la pensée peut accéder à l'être, et non pas le projet d'une métaphysique comme ontologie. Les textes introductifs de la Logique indiquent clairement que l'usage du concept de métaphysique est ici avant tout polémique et dirigé contre "le renoncement au penser spéculatif " ${ }^{17}$ dont sont responsables tout aussi bien un empirisme diffus que la philosophie kantienne : « la science et le sens commun se renforçant ainsi l'un l'autre pour provoquer le déclin de la métaphysique ${ }^{18}$. Métaphysique ne signifie ici ni discipline ni projet théorique déterminé, mais désigne seulement l'une des caractéristiques des métaphysiques passées, la prétention à saisir l'être par la pensée : «l'ancienne métaphysique avait à propos du penser un concept plus élevé que celui qui est devenu monnaie courante à l'époque moderne. Elle posait en effet comme fondement que seul est véritablement vrai en les choses ce qui est connu d'elles et en elles par la pensée ${ }^{19}$ ". Reste à déterminer le sens du projet d'une transformation de la métaphysique où la logique transcendan-

13 Critique de la raison pure, T.P., PUF, p. 222 : « le titre pompeux d'une ontologie, qui prétend donner, des choses en général, une connaissance synthétique a priori dans une doctrine systématique, doit faire place au titre modeste d'une simple analytique de l'entendement pur ".

14 Log. I, p. 21.

15 Ibid., p. 16.

16 Enc., § 24.

17 Log. I, p. 2.

18 Ibid., p. 3.

19 Ibid., p. 13. 
tale " prend la place de la métaphysique d'autrefois ${ }^{20}$ ". Doit-il être compris comme une tentative de restauration de la métaphysique pré-critique, ou comme une rupture avec cette métaphysique?

Par "ancienne métaphysique ", Hegel entend avant tout la métaphysique scolaire ou ce qui est présenté à propos de Kant comme « l'état de la métaphysique de son temps " ${ }^{21}$ Il s'agit bien d'une forme de métaphysique déterminée, et l'on pourrait penser que ce qui en est dit ne peut concerner l'ensemble des métaphysiques passées. Néanmoins, il est significatif que lorsque Hegel oppose ces métaphysiques à des pensées philosophiques plus profondes (comme celles d'Aristote à propos de l'âme), il évite soigneusement de les désigner comme des métaphysiques et préfere les donner en exemple d'une pensée authentiquement "spéculative " ${ }^{22}$. Tout semble indiquer que l'état de l'ancienne métaphysique est révélateur des défauts généraux de ce qu'il considère comme le style métaphysique en philosophie sous lequel il range également la Scolastique ${ }^{23}$ C'est dans le Concept préliminaire que l'on trouve la présentation la plus claire des critiques qui lui sont adressées. La métaphysique y est définie comme " la démarche naïve qui renferme la croyance que, grâce à la réflexion, la vérité est connue ${ }^{24}$ La métaphysique tente de saisir la vérité par la pure pensée tout en présupposant que la pensée pure à accès aux vérités les plus hautes, que « seul est véritablement vrai en les choses ce qui est connu d'elles et en elles au moyen de la pensée ${ }^{25}$ Cette entreprise philosophique n'est pas critiquée en ce qu'elle prétend que la pensée est capable de saisir le vrai, mais pour son caractère "naif " ${ }^{26}$. Hegel lui reproche fondamentalement de prétendre saisir la vérité par la pensée sans penser ni les pensées qu'elle met en œuvre dans cette entreprise ni la capacité de sa pensée à saisir l'être, et en ce sens il reprend à son compte la critique kantienne du dogmatisme métaphysique. Quant au contenu, le discours métaphysique est à la recherche

20 Ibid., p. 37.

21 Log. III, p. 306.

22 Ibid., p. 308 ("les idées vraiment spéculatives d'Aristote ").

23 Enc., $§ 36$, add., pp. 492-493 : “Au lieu de parvenir à l'identité concrète, cette métaphysique persistait dans l'identité abstraite; mais ce qu'elle eut de bien, c'est la conscience que la pensée seule est l'essentialité de l'étant. Les philosophes anciens et notamment les Scolastiques fournirent la matière pour cette métaphysique. Dans la philosophie spéculative, l'entendement est bien un moment, mais un moment auquel on n'en reste pas. Platon n'est pas un métaphysicien de ce genre, et Aristote encore moins, quoique l'on croie habituellement le contraire. "Pour une comparaison de la métaphysique et de la scolastique, Enc., § 38, add., pp. 495-496.

24 Ibid., § 26.

25 Log. I, p. 13.

26 Enc., $\S \S 26,27$ et 41 add. 1. 
des prédicats du vrai, des prédicats qui définissent l'essence de l'être en général (pour l'ontologie) de l'âme, du monde ou de Dieu (pour les différentes branches de la métaphysique spéciale). Hegel reproche alors à la métaphysique d'attribuer des prédicats à un objet sans s'interroger sur le contenu logique de ces prédicats, sans penser ce qui est pensé par ces prédicats (la réalité, l'infinité, etc.), et sans s'interroger sur la compatibilité de ces prédicats et des sujets auxquels ils sont attribués ${ }^{27}$ Quant à la forme, la naïveté caractéristique du discours métaphysique tient à l'absence de mise en question de la domination des schèmes de la Logique formelle ${ }^{28}$ Hegel reproche alors à la métaphysique de ne pas s'interroger sur la question de la validité de la forme prédicative, de ne pas poser la question de savoir si le jugement est la forme logique la plus à même d'exprimer le vrai ${ }^{29}$ L'un des objectifs de la Logique sera précisément de soumettre les pensées de l'ancienne métaphysique à l'examen critique qui leur fait défaut, en leur appliquant une opération dont Hegel dit lui-même qu'elle est analogue à celle que Kant a fait subir à la métaphysique ${ }^{30}$ Il s'agira de soumettre différentes pensées à un examen destiné à vérifier dans quelle mesure elles sont susceptibles d'exprimer le vrai, aussi bien les différents prédicats pouvant être identifiés à l'objet de la métaphysique (Logique objective) que les différentes formes du discours rationnel (Logique subjective).

Pour comprendre quelles transformations de la métaphysique résultent de cette critique de la métaphysique, repartons des structurations de la métaphysique scolaire en une métaphysique générale (ou ontologie) et en une métaphysique spéciale (psychologie, cosmologie et théologie rationnelles). Hegel indique que sa Science de la logique remplace l'intégralité de la métaphysique : la métaphysique générale et la métaphysique spéciale sont contenues dans la Logique objective ${ }^{31}$. Mais ce qui frappe au premier abord, c'est que la Doctrine de l'être et la Doctrine de l'essence ne peuvent à proprement parler constituer ni l'une ni l'autre car elles n'en conservent ni les objets ni les fonctions.

27 Enc., §§ 28-29; voir également Log. III, pp. 247-248.

28 On pourrait rapprocher ce thème de la critique heideggérienne de la domination de la logique caractéristique de la métaphysique occidentale (voir à ce propos, F. Dastur, « La destruction heideggéreinne de la logique ", in F. Dastur, C. Lévy, Etudes de philosophie ancienne et de phénoménologie, L'Harmattan, pp. 335-356). F. Dastur voit dans la Phénoménologie de l'esprit une tentative de soustraire la pensée à la domination de la logique et dans la Logique un retour à la métaphysique, sans voir l'enjeu critique de la thèse suivant laquelle la transformation de la métaphysique en logique doit s'accompagner d'une transformation de la logique ("Phénoménologie, logique, ontologie de Lambert à Hegel ", ibid., pp. 181-213).

29 Enc., § 28.

30 Log. I, p. 37.

31 Log. I, p. 37. 
L'objet propre de l'ontologie est l'être en tant qu'être, ou l'objet en général, c'est-à-dire les caractéristiques générales des objets en tant que chose en soi. Or, le fait que la Science de la logique soit précédée d'une Phénoménologie de l'esprit indique assez clairement que l'être y est considéré tel qu'il est pour la conscience, ou plus précisément, pour la pensée ${ }^{32}$ En tant que la Science de la logique procède à l'étude critique de l'ensemble des prédicats pouvant prétendre à fournir une réponse à la question " qu'est-ce que l'être ", elle prend la place de l'ontologie tout en déplaçant l'objet de l'ontologie puisqu'elle ne se présente pas comme une étude de l'être lui-même, mais de la pensée métaphysique de l'être. Un des traits les plus remarquables de cette métaphysique transformée est qu'elle consiste en une pensée de la métaphysique, en une pensée des pensées métaphysiques, en une "méta-métaphysique " ${ }^{33}$ La Science de la logique ne respecte pas non plus la fonction de l'ontologie : élaborer la science première dont les métaphysiques spéciales ne seront que des spécifications. Insistons à ce propos sur le fait que le système hégélien ne reproduit pas en son sein l'architecture hiérarchique qui subsumerait une étude des régions de l'être (Philosophie de la nature et de l'esprit) sous une étude de l'être en général (Logique). D'après Hegel, la succession des parties de l'Encyclopédie n'est pas l'application de l'idée absolue à différents objets mais «l'exposition " d'un même objet («l'idée elle-même") en trois "éléments divers $»^{34}$. Cette succession n'est pas conçue par Hegel comme une subsomption mais comme un développement et si la première partie peut effectivement prétendre à une généralité qui fait défaut aux deux dernières, elle ne se rapporte pas pour autant à elles comme un genre à des espèces puisque ce qui définit la Philosophie de la nature, c'est l'aliénation de l'idée, ou le fait que les rapports ontologiques qui caractérisent la nature contredisent au moins partiellement les propositions conclusives de la Logique.

Mais il n'en résulte pas pour autant que la Logique n'ait aucune signification ontologique ${ }^{35}$ Hegel y montre qu'aucun des prédicats qui sont attribués à l'être par la métaphysique ne peut fournir une réponse satisfaisante à la réponse "qu'est-ce que l'être ». Il y montre également que tous ces prédicats ont une part de vérité, de sorte que la réponse à la question "qu'est-ce que l'être " ne peut être formulée en un jugement, en une proposition attribuant un prédicat déterminé à un sujet, mais

32 Voir à ce propos, H.-F. Fulda, « Die ontologie und ihr Schicksal in der Philosophie Hegels. Kantkritik in Fortsetzung kantischer Gedanken ", in Revue internationale de Philosophie, 4/1999, n 210, pp. $465-483$.

33 H.-F. Fulda, "Spekulativ Logik als die " eigentliche Metaphysik ". Zu Hegels Verwandlung des neuzeitlichen Metaphysikverständnisses ", in D. Pätzold, A. Vanderjagt (eds.), Hegels Transformation der Metaphysik, Jürgen Dinter Verlag für Philosophie, Köln, 1991, pp. 9-27, ici p. 20.

34 Enc., § 18, Rq. Voir à ce propos, H. F. Fulda, ibid., pp. 15-18.

35 Contrairement à ce que soutient $\mathrm{H}$.-F. Fulda dans les articles cités. 
doit prendre la forme de l'examen critique de tous les prédicats. C'est en ce sens que la Logique subjective est la vérité de la Logique objective et qu'elle s'achève sur l'idée absolue, ou idée logique, qui se voit elle-même définie comme une méthode. La réponse à la question " qu'est-ce que l'être " est une méthode parce qu'il n'y a pas de réponse particulière (prédicative) possible, et parce que la seule réponse possible est l'examen critique et méthodique de toutes les réponses possibles. Néanmoins, Hegel soutient bien que l'idée logique "est la vérité absolue et toute la vérité ", "l'unité absolue du concept et de l'objectivité " ${ }^{36}$, qu'elle seule est "être, vie non-caduque, vérité se sachant, et toute vérité " ${ }^{37}$ L'idée logique n'est pas présentée ainsi comme un simple mouvement méthodique du discours, mais également comme le mouvement de l'être lui-même, ce qui lui confere bien une portée ontologique : dire de l'être qu'il est l'idée, c'est dire qu'il est développement d'une intériorité, d'une négativité et d'une totalité.

En définitive, le fait qu'elle soit présentée comme une méthode (dans une Logique subjective ne correspondant pas à proprement parler, d'après Hegel luimême, à la métaphysique) indique seulement que l'objectif de Hegel n'est pas tant de produire des énoncés ontologiques qu'une démarche permettant de justifier ou infirmer des thèses à portée ontologique, que celles-ci relèvent d'une métaphysique générale, d'une métaphysique spéciale ou de ce que nous appellerons une «ontologie régionale ». Dans la Logique, les prédicats métaphysiques ne se voient reconnus qu'une vérité partielle en tant que réponses à la question de la métaphysique générale, mais le fait qu'ils aient une vérité partielle les autorise également à prétendre à une vérité dans tels ou tels secteurs de l'être, en tant que réponse aux questions des métaphysiques spéciales ou régionales. Cependant, quel que soit le point de vue métaphysique adopté (général ou régional), la Logique ne pourra produire que des arguments en faveur de telle ou telle thèse à portée ontologique sans pour autant en établir par elle-même la vérité. $D$ 'une part, la conclusion ontologique de la méta-métaphysique de l'idée devra elle-même être "vérifiée " ${ }^{38}$ dans les autres parties du système. Si la Science de la logique est bien la science fondamentale, elle n'est pas la science première mais tout autant " science philosophique dernière " ${ }^{39}$ puisque c'est seulement au terme du développement encyclopédique que sa vérité est définitivement établie. D'autre part, les thèses relevant d'une métaphysique spéciale ou régionale ne pourront être tranchées

36 Enc., $\S \S 236$ et 213.

37 Log. III, p. 368.

38 Enc., § 574.

39 H.-F. Fulda, ibid., p. 20. 
par des décrets de la raison pure, mais seulement dans l'explicitation du contenu logique des différents savoirs portant sur les différentes régions de l'être.

Concluons donc que si Hegel entend reprendre à son compte le projet métaphysique, c'est en en restreignant considérablement l'importance et sous une forme très modifiée. Chez un penseur comme Kant, la notion de métaphysique circonscrit l'intégralité de l'activité philosophique alors que chez Hegel, elle ne désigne plus que les deux premières parties de la première partie du système (Logique objective). On a vu par ailleurs que la métaphysique y est transformée en une méta-métaphysique qui rompt avec son objet et sa méthode caractéristique. Plutôt qu'à un retour à la métaphysique passée, nous avons bien affaire à une rupture qui va de pair avec une redéfinition de la philosophie comme forme de pensée non métaphysique. Tel est précisément l'un des enjeux de la redéfinition de la philosophie comme science.

Si Hegel revendique la scientificité de la philosophie, c'est certes parce qu'il prétend qu'elle doit remplir une fonction fondatrice. La spéculation prétend fonder l'ensemble des savoirs, y compris les savoirs scientifiques, et en ce sens, il est légitime qu'elle prétende à la scientificité et même à la scientificité la plus haute. Cette revendication est assez classique, mais le projet hégélien d'une science philosophique est également solidaire d'une interprétation bien particulière de la fondation du savoir.

Hegel ne tente aucunement en effet de procéder à cette fondation à la manière des systèmes métaphysiques du XVII ${ }^{\mathrm{e}}$ siècle qui prétendent tout à la fois intégrer l'ensemble du savoir scientifique et le dériver de principes premiers. La préface de la Phénoménologie de l'esprit contient une célèbre critique de l'idée de principe, et dans ses Leçons sur l'histoire de la philosophie, Hegel dénonce la conception anachronique de la science que présuppose la théorie fichtéenne du premier principe : "C'est une philosophie tout d'une pièce que Fichte a tenté de faire, une philosophie dans laquelle rien d'empirique ne serait reçu de l'extérieur. Mais ce faisant, un point de vue faux est aussitôt introduit et cette pensée ressortit à l'ancienne représentation que l'on avait de la science et qui consistait à commencer sous cette forme par des principes " ${ }^{40} \mathrm{La}$ philosophie spéculative ne prétend pas non plus intégrer en elle un savoir positif dont elle reconnaît l'indépendance et ne tente de déduire que la vérité. C'est en ce sens que la Phénoménologie de l'esprit soutient que transformer la philosophie en science revient à produire une exposition, et non une production, du vrai, à exposer le vrai sous la forme de la véritét $e^{41}$ C'est en ce sens également que les textes introductifs de la Philosophie de la nature déclarent que ce qui distingue le savoir philosophique et le savoir scientifique n'est pas tant leur contenu que la manière dont ce contenu est ex-

40 Leçons sur l'histoire de la philosophie, t. 7, Vrin, 1994, p. 1981.

41 Préface, p. 39 : « La vraie forme dans laquelle la vérité existe ne peut-être que le système scientifique de celle-ci." 

position d'un contenu produit indépendamment d'elle. Enfin, Fichte soutient que la certitude que le premier principe soit effectivement un premier principe repose sur sa capacité à épuiser l'intégralité du savoir dans le cadre d'une déduction exhaustive ${ }^{47}$ On retrouve également ce thème de la circularité de la science systématique dans l'Encyclopédie où la science fondamentale qu'est la Logique ne voit sa vérité confirmée qu'une fois effectuée l'ensemble de la déduction du savoir constitué indépendamment de la philosophie. Ajoutons que chez Fichte déjà, cette définition de la scientificité était indissociable d'une critique de la métaphysique. Par métaphysique, Fichte entend parfois le projet de produire des connaissances par la seule pensée, alors que la science se contentera d'une "exposition " ${ }^{48}$, ou d'une "reconstruction " ${ }^{49}$ du savoir produit indépendamment de la conscience par la philosophie. À ce propos, c'est sans doute dans les textes rédigés à l'occasion de la querelle de l'athéisme que se trouvent les formulations les plus claires : "dans la mesure où la métaphysique doit être le système des connaissances réelles, produites par le simple penser, Kant - et moi avec lui - nie totalement la possibilité de la métaphysique (...) Dans la mesure où notre système rejette les extensions pratiquées par les autres systèmes, il ne lui vient pas à l'esprit, de vouloir étendre le penser commun, qui est le seul penser réel; mais il veut uniquement l'embrasser et l'exposer exhaustivement " $"$ Hegel reprendra à son compte la définition fichtéenne de la philosophie comme exposition et " reconstruction " ${ }^{51}$, il reprendra également à son compte la critique de la métaphysique qui en résulte.

Si la philosophie ne se rapporte pas aux savoirs positifs comme à une métaphysique surplombante, quels rapports entretient-elle avec eux ? Hegel l'explique dans les additifs introductifs de la philosophie de la nature. La Naturphilosophie y est présentée comme une tentative de fondation spéculative (fondée sur l'analyse du contenu logique des différentes pensées scientifiques) des différents universels produits par les sciences de la nature. Ces universels consistent d'une part, en ce que l'on peut appeler des principes définitionnels, formulés dans la définition de concepts tels le concept

47 Fichte, Essais, pp. 47-51.

48 Fichte, Essais, p. 64.

49 Fichte, Rapport clair comme le jour, Vrin, 1986, pp. 52 et 56-57.

50 Fichte, "Rappels, réponses, questions", op. cit, p. 138. Fichte donne parfois d'autres significations à la notion de métaphysique, voir par exemple le début du $\S 6$ de la Grundlage et la préface de la seconde édition de Sur le concept de la doctrine de la science.

51 W. 5, p. 30. 
d'espace, d'électricité, de substance chimique, d'autre part en ce que l'on peut appeler des principes explicatifs, principes faisant intervenir des concepts comme le concept de force d'attraction, d'affinité chimique, enfin, ils consistent en des lois. Crucial pour notre propos est le fait que Hegel fasse également entrer sous ces universels ce qu'il appelle la métaphysique d'une science ou d'un concept scientifique ${ }^{52}$. Le manuscrit des Leçons de 1822/1823 contient l'affirmation selon laquelle : "La physique a une métaphysique consciente ou inconsciente " ${ }^{53}$. Quant à l'additif du paragraphe 248 de l'Encyclopédie, il conclut un développement consacré aux sciences en remarquant que "toute conscience cultivée a sa métaphysique " ${ }^{54}$ Que désigne métaphysique ici ? Les déterminations de pensée les plus générales et les plus fondamentales (" les catégories de la physique ") qui configurent le savoir en agissant comme un "instinct pensant " et une "puissance absolue " 55 Il est dit à ce propos : " métaphysique ne signifie rien d'autre que l'étendue des déterminations de pensée universelles, en quelque sorte un filet de diamant dans lequel nous mettons toutes choses et par lequel seulement nous rendons intelligible " ${ }^{56}$. Que l'explicitation de l'action des structures conceptuelles sur la pensée soit l'objet de la logique, c'est un thème sur lequel avait déjà insisté la préface de la seconde édition de la Science de la logique ${ }^{57}$ Les concepts dont il est question sous le nom de "catégories de la physique " sont les concepts les plus généraux qui sont à l'œuvre dans le savoir en agissant comme son " configurateur intérieur " ${ }^{58}$; ils ont déjà été étudiés une première fois dans la Logique car leur généralité leur conferent précisément une prétention métaphysique qui justifie qu'il en soit ici question comme d'une métaphysique. Mais la notion est prise ici en un sens plus précis encore. Ces concepts sont en effet eux-même porteurs d'une métaphysique (métaphysique d'un concept) au sens où ils constitutifs des différentes ontologies spéciales auxquelles se

52 Pour la métaphysique d'un concept : Log. I, p. 237 : « la mathématique ne peut tirer au clair ce qu’il en va de la métaphysique de son propre concept [d'infini] ". Le sens du concept de métaphysique des sciences a été peu étudié et le commentaire tend à minimiser l'originalité de la position hégélienne sur cette question, soit en, réduisant les principes métaphysiques hégéliens aux principes métaphysiques au sens de Kant (c'est l'hypothèse qui commande l'ouvrage de B. Falkenburg, Die Form der Materie), soit en soutenant que contrairement à Kant, qui n'attribue à ces principes qu'un rôle heuristique, Hegel conçoit la métaphysique comme génératrice de la vérité physique (G. Buchdahl, "Hegel's Philosophy of Nature and the Structure of Science ", op. cit., p. 6).

53 VG, p. 73. Les leçons de 1821/1822 précisaient " une métaphysique complète " (Vorlesungen über Naturphilosophie. Berlin 1821/1822, Peter Lang, 2002, p. 2).

54 Enc., § 246, add., W. 9, p. 20.

55 VG, p. 73.

56 Enc., § 246, add., W. 9, p. 20, voir aussi W. 18, p. 77 et VG, p. 73.

57 W. 5, pp. 26-27, 30.

58 Log III, p. 56. 
réferent les sciences : la catégorie d'atome définit une variante de la métaphysique mécaniste dominant en Mécanique ${ }^{59}$, les concepts de force et d'opposition définissent le dynamisme dominant dans une partie des Sciences physiques ${ }^{60} \mathrm{Si}$ ces concepts, qui structurent les savoirs particuliers et qui sont à l'œuvre dans toute conscience, sont ici identifiés à une métaphysique, c'est donc également parce qu'ils définissent différentes ontologies que la science spéculative pourra reconnaître comme des métaphysiques spéciales légitimes ou illégitimes relativement aux objets auxquels elles sont appliquées.

Certains commentateurs ont soutenu que l'objectif de Hegel était d'extirper la métaphysique des sciences ${ }^{61}$ Une telle interprétation semble démentie par les textes. Hegel soutient en effet, que les scientifiques se trompent quand ils croient pouvoir se passer de métaphysique. Dans le manuscrit des Leçons de 1822/1823, l'occasion est ainsi fournie de comparer à ce propos Newton à M. Jourdain : " Newton refusait la métaphysique, il me fait penser à ce paysan à qui l'on a dit qu'il parlait en prose et qui était fier de le raconter chez lui à sa femme " ${ }^{62}$. Dans les Leçons sur l'histoire de la philosophie, il considère comme un défaut général de l'empirisme le fait de croire pouvoir se passer de métaphysique dans la description de l'expérience ${ }^{63}$ Hegel soutient néanmoins que la métaphysique des sciences doit toujours faire l'objet d'une critique. Les textes introductifs indiquent que ce qui dans les sciences est non satisfaisant, c'est avant tout la métaphysique des sciences, et plus précisément, la forme ou la manière ("die Weise der Metaphysik») qui est donnée à cette métaphysique, forme dont Hegel dit qu'elle a déjà été critiquée dans la Logique ${ }^{64}$. En quoi la métaphysique des sciences est-elle inadéquate? S'il y a toujours métaphysique dans une science, la question est seulement de savoir si elle est appropriée ou pas à

59 Enc., § 98, add. 1, p. 531.

60 Enc., $\$ 98$, Rq., et W. 5, p. 21.

61 Voir par exemple K. N. Ihmig, "Hegel's Treatment of Universal Gravitation", in M. J. Petry, Hegel and Newtonianism, Kluwer Academic Publishers, 1993, pp. 367-381, ici p. 373.

62 VG, p. 37.

63 Hist. Phi., t. 6, pp. 1280-1281 : "L'autre défaut formel commun à tous les empiriques est qu'ils croient s'en tenir à l'expérience; que dans l'accueil des perceptions ils fasse de la métaphysique, ils en demeurent inconscients ".

64 V.G., pp. 67 et 73; Enc., $§ 246$ add., W. 9, p. 20 ; Théorie de la mesure [cité Mesure], trad. A. Doz, PUF, 1970, p. 67. 
l'objet de cette science ${ }^{65}$ On constate en effet que l'objet des critiques hégéliennes est toujours l'inadéquation des principes métaphysiques aux autres principes d'une science et que cette inadéquation peut prendre deux formes. D'une part, la critique peut s'en prendre au réductionnisme scientifique, c'est le cas lorsque Hegel dénonce la tendance à appliquer les principes d'une discipline à d'autres domaines qui se définissent pourtant par des rapports ontologiques différents; c'est de ce point de vue qu'il critique l'optique newtonienne ${ }^{66}$, l'électromagnétisme naissant ${ }^{67}$ et l'électrochimisme ${ }^{68}$ D'autre part, la critique peut concerner un simple désajustement, au sein d'une même théorie, entre les principes métaphysiques et les autres concepts de la science. Un tel désajustement peut être observé dans le cas de la mécanique newtonienne qui, en admettant une métaphysique mécaniste plutôt qu'une métaphysique dynamiste, est conduite à une explicitation inadéquate de la nature des forces physiques, et par là même, à une explicitation inadéquate des principaux concepts de la mécanique $^{69}$ Ainsi Hegel, pour qui les hypothèses mécanistes interdisent une compréhension véritable de la nature de la gravité, se demande-t-il : "Quand donc la science en viendra-t-elle enfin à obtenir la conscience des déterminations métaphysiques qu'elle utilise, et a prendre pour fondement, à leur place, le concept de la chose $"{ }^{70}$ On peut également observer un tel désajustement dans le cas de la théorie chimique de Berzélius qui tente de donner une explication atomiste des phénomènes chimiques alors que les concepts fondamentaux de la chimie ne peuvent être explicités adéquatement qu'en termes dynamistes. À propos de Berzélius, Hegel écrit : " cette métaphysique dénuée de fondement n'a rien à voir avec les proportions de la saturation elle-même ", c'est-à-dire avec la théorie des proportions définies qu'elle est pourtant censée fonder ${ }^{71}$

65 Enc., § 98, add. À l'injonction newtonienne suivant laquelle les physiciens doivent se garder de la métaphysique, Hegel répond : "De purs et simples physiciens, seuls le sont en fait des animaux, étant donné qu'eux ne pensent pas, alors que l'homme, en tant qu'être pensant, est un métaphysicien-né. Ce qui importe alors seulement, c'est si la métaphysique que l'on utilise a la nature qui convient, et notamment si ce ne sont pas, à la place de l'idée logique concrète, des déterminations de pensées unilatérales, fixées par l'entendement, qui forment ce à quoi l'on s'en tient, et qui constituent le fondement de notre activité théorique " (p. 531).

66 Enc., § 276, Rq. et add.

67 Enc., § 246, add., W. 9, p. 20

68 Enc., § 330, Rq.

69 Que la critique de la mécanique newtonienne ne consiste qu'en cette critique modérée, et non en une pure et simple réfutation, c'est ce que nous avons tenté de montrer dans notre Hegel. La naturalisation de la dialectique, Vrin, 2001, pp. 210 sq.

70 Enc., § 271, Rq., W. 9, p. 89.

71 Mesure, p. 69. 
Le défaut principal de la métaphysique des sciences de la nature est donc qu'elle conduit à penser le savoir sous des prédicats métaphysiques incompatibles avec ce qui est pensé par les autres principes des sciences. Ce défaut se ramène au défaut général de l'ancienne métaphysique puisque dans les deux cas, on use des prédicats métaphysiques sans véritablement penser leur contenu et la légitimité de leur usage. Dans le Concept préliminaire, à propos de l'empirisme, quelques paragraphes après avoir critiqué l'ancienne métaphysique, Hegel adresse les mêmes reproches à la science empirique : "l'illusion principale de l'empirisme scientifique est donc toujours celle-ci : (...) il ne sait pas qu'il contient lui-même et applique une métaphysique, qu'il utilise ces catégories et leurs liaisons de manière totalement non critique et inconsciente " 72

Quel est donc le sens épistémologique de la thèse suivant laquelle toute science a sa métaphysique? L'affirmation suivant laquelle il y a de la métaphysique dans les sciences pourrait paraitre paradoxale, dans la mesure où l'on a coutume depuis Comte, d'opposer science et métaphysique et que l'on peut faire remonter cette opposition à Bacon et à Newton ${ }^{73}$ L'opposition de la science et de la métaphysique appartient donc à une tradition bien établie dès l'époque de Hegel, mais la thèse suivant laquelle toute science à sa métaphysique renvoie elle aussi à une tradition établie. À la fin du XVIII ${ }^{\mathrm{e}}$, l'idée d'une métaphysique des sciences pouvait être entendue en deux sens. En un sens strict et normatif, et en un sens large et descriptif.

C'est en ce premier sens, strict et normatif, qu'il est question chez Kant des Premiers principes métaphysiques de la science de la nature. L'idée suivant laquelle les sciences doivent être fondées sur des principes métaphysiques appartient à la tradition rationaliste qui prend sa source chez Descartes et Leibniz. D'après ces philosophes, les sciences ne peuvent être fondées que si elles sont dérivables des principes de l'ontologie générale (moyennant la spécification caractéristique de la métaphysique spéciale). C'est de cette tradition que Kant hérite lorsqu'il se met en quête des principes métaphysiques de la science de la nature, même si chez lui, la notion de métaphysique ne désigne plus que les principes qui ont leur sources dans la raison pure. Au cours du XVIII ${ }^{e}$ siècle, l'idée de principes métaphysiques a également été étendue au sens descriptif des présuppositions générales concernant la réalité étudiée par une science. C'est en ce sens que d'Alembert, dans les Éléments de philosophie, soutient que toute

72 Enc., § 38, Rq.

73 On peut remarquer que la formule de Newton : " Physique garde-toi de la métaphysique! ", est rapportée à la fois par Comte et Hegel, et que tous deux la jugent contradictoire, (Cours de philosophie positive, Hermann, Paris, 1975, t. 1, p. 531 ; Hist. Phi., t. 6, p. 1573), mais pour des raisons opposées; Comte parce qu'il juge que Newton est resté trop métaphysicien, Hegel parce qu'il reproche à Newton de n'avoir pas été métaphysicien de façon convaincante et réfléchie. 
science a sa métaphysique ${ }^{74}$, et que L. Carnot parle de la métaphysique du calcul infinitésimal ${ }^{75}$ On notera qu'entendus en ce sens descriptif, les principes métaphysiques ne désignent plus ce que la vérité d'une science présuppose, mais seulement un certain type de propositions générales contenu dans une science. On notera également que la notion de métaphysique est ici dotée d'une extension plus grande que dans le premier sens. Elle désigne toujours ce qui relève de la métaphysique générale (ainsi d'Alembert nomme-t-il principe métaphysique le principe de causalité ou de raison suffisant $\mathrm{e}^{76}$ ) voire de la spécification de ces principes généraux par la nature particulière de l'objet (métaphysique spéciale), mais elle désigne aussi les rapports " ontologiques " propres à une science, qui sont considérés pour eux-mêmes et indépendamment de toute spécification d'une métaphysique générale (on parlera donc à ce propos d'" ontologie régionale »).

Il semble en fait que Hegel entende la notion de principes métaphysiques suivant ces deux acceptions à la fois. Il prétend en effet rapporter les principes scientifiques aux catégories générales qui permettent de rendre compte de leur rationalité. En ce sens, la fondation des sciences de la nature ne dépend pas seulement de la Naturphilosophie, mais aussi de la Logique, plus précisément, de la Logique objective est tant qu'elle tient lieu de métaphysique générale (sans pour autant en constituer une). Conjointement, Hegel entend l'idée de métaphysique des sciences en un sens descriptif, au sens de la métaphysique produite par une science de la nature; elle peut être inadéquate aux autres principes de la science en question et donc avec l'ontologie régionale dont la spéculation entend montrer qu'elle définit un niveau déterminé de la nature. Puisque ces ontologies régionales sont élaborées à partir de l'universel produit par le savoir positif (les principes explicatifs et définitionnels dont dépend par exemple l'organisation et la rationalité d'une science de la nature), on voit qu'il ne s'agit pas ici de faire entrer les sciences sous le giron d'une métaphysique spéciale, mais seulement de proposer un argument en faveur de thèses à portée ontologique.

74 Éclaircissements sur les différents endroits des Éléments de philosophie, in Essai sur les éléments de philosophie, Fayard, Paris, 1986, p. 348 : "À proprement parler, il n'y a point de science qui n'ait sa métaphysique, si l'on entend par là les principes généraux sur lesquels la science est appuyée, et qui sont comme le germe des vérités de détail qu'elle renferme et qu'elle expose; principes d'où il faut partir pour découvrir de nouvelles vérités, ou auxquels il est nécessaire de remonter pour mettre au creuset les vérités qu'on croit découvrir ". D'Alembert restreint cependant l'universalité des principes métaphysiques, en affirmant qu'on ne peut appliquer le mot métaphysique qu'aux sciences ayant des objets immatériels, c'est-à-dire les mathématiques et la physique générale (pp. 348-349).

75 Dans ses Réflexions sur la métaphysique du calcul infinitésimal (1797), L. Carnot entend alors par métaphysique «le véritable esprit de l'Analyse infinitésimale " (p. 1), ou l'analyse infinitésimale saisie d'après ses principes (p. 4).

76 Traité de dynamique (1758), Edition Jacques Gabay, Réimpressions, 1990, p. XXIII. 
A propos de ces ontologies régionales, Hegel combine donc les différents usages possibles de l'idée de métaphysique des sciences en parvenant ainsi à formuler une problématique originale, celle de l'adéquation des principes métaphysiques aux autres principes d'une science, celle des contradictions et des obstacles (la métaphysique d'une science formant " ce à quoi l'on s'en tient " ${ }^{77}$ ) qui en résultent. La question spécifiquement hégélienne concerne la conformité des principes métaphysiques aux autres principes de la science en question car les principes métaphysiques peuvent conduire à une explicitation inadéquate des autres principes d'une science et en masquer la rationalité, de sorte que la Philosophie de la nature ne pourra se contenter d'expliciter le savoir scientifique et devra parfois polémiquer avec lui. Par ces polémiques, la philosophie ne s'en prend pas à l'indépendance des sciences, mais traite seulement de ce qui, malgré cette indépendance, continue de relever en elles de la philosophie. 